\title{
Quale traducente per empowerment?
}

\author{
Claudio Giovanardi
}

PUBBLICATO: 22 MARZO 2019

\begin{abstract}
Quesito:
Alcuni lettori ci chiedono se non sia possibile proporre un'alternativa italiana, sul modello dello spagnolo che ha adottato empoderamiento, al termine inglese empowerment. Questa voce è presente anche nella comunicazione scolastica: "esercitare una leadership come empowerment" rientra, ci scrive un lettore, tra le funzioni del dirigente.
\end{abstract}

\section{Quale traducente per empowerment?}

Digitando empowerment in Google si ottengono circa ro4 milioni di risultati. Questo semplice dato numerico è significativo della diffusione amplissima del termine inglese. Uno dei criteri fondamentali che sono alla base del successo di un traducente italiano di qualsivoglia parola straniera è legato al livello di penetrazione del forestierismo; non solo in termini quantitativi, ma soprattutto per ciò che attiene alla sua capacità di irradiamento nei settori della conoscenza. Da questo punto di vista empowerment, vocabolo già circolante negli anni Novanta, ma affermatosi nei primi anni Duemila, è diffuso in almeno due importanti linguaggi settoriali: quello economico-finanziario e quello medico-psicologico, all'interno dei quali assume significati peculiari, rendendo molto difficile un'ipotetica reductio ad unum. Individuare un unico traducente italiano che possa riassumere in sé l'intero spettro semantico dell'anglicismo è probabilmente impossibile. Del resto, la World Health Organization ha faticato molto, nel 20I2, a elaborare una definizione di empowerment che fosse in grado di saldare i diversi valori del termine nel campo della medicina, della psicologia e della psicoanalisi.

In Wikipedia troviamo la seguente definizione di empowerment: "un processo di crescita, sia dell'individuo sia del gruppo, basato sull'incremento della stima di sé, dell'autoefficacia e dell'autodeterminazione per far emergere risorse latenti e portare l'individuo ad appropriarsi consapevolmente del suo potenziale". Quando il riferimento è all'individuo, si parla più propriamente di self empowerment, termine per il quale la traduzione letterale sarebbe autopotenziamento, che in effetti conosce un uso (in particolare nel campo delle neuroscienze) sia pur limitato (le occorrenze in Google sono appena 19.300). In altri contesti, tuttavia, occorrerebbero altri corrispondenti italiani, come autolegittimazione, emancipazione, autodeterminazione, ecc.

Rispetto al quesito posto dal lettore che ci segnala l'uso nel Piano di offerta formativa di un liceo, tuttavia, occorre fare una precisazione. La difficoltà di traduzione di un termine tecnico-scientifico, or ora denunciata per empowerment, non può essere tirata in ballo quando di quel termine si fa un uso improprio e, tutto sommato, inutile. Nell'esempio riportato, siamo di fronte a un documento scolastico relativo alle prerogative del Dirigente scolastico. In quel contesto non vi è alcuna necessità di usare l'anglicismo empowerment, che peraltro risulta piuttosto oscuro; sarebbe bastato sostituirlo con (azione di) valorizzazione o (azione di) mighioramento per render ragione dell'intento comunicativo dello scrivente. Purtroppo la decontestualizzazione del forestierismo e il suo uso al posto di tanti ottimi corrispondenti italiani rappresentano il vero problema con cui dobbiamo misurarci quotidianamente.

\section{Cita come:}

Claudio Giovanardi, Quale traducente per empowerment?, "Italiano digitale", 2019, VIII, 2019/1 (gennaio-marzo), pp. 50.

DOI: $10.35948 / 2532-9006 / 2019.3079$ 